\title{
La música y la personalidad: factores sociodemográficos y culturales relacionados
}

\section{The music and personality: sociodemographic and cultural factors related}

\author{
Patricia Martínez ${ }^{1 *}$, Hugo Torres ${ }^{1}$ \\ 1 Universidad Anáhuac México Norte. Huixquilucan, México.
}

\section{Resumen}

El presente trabajo examina la relación entre la música y su elección como vocación profesional y factores de personalidad, culturales y sociodemográficos. Participaron 86 sujetos de distintas áreas de estudio (Música, Psicología, Ingeniería y Medicina). En todas las áreas de medición (depresión, tendencia suicida, ansiedad, paranoia, obsesivo-compulsivo, esquizofrenia y adicciones), los estudiantes de música mostraron ser más propensos por cerca de $10 \%$ de superioridad sobre los demás estudiantes. De esta forma, mediante una serie de correlaciones, se demostró que existen rasgos de personalidad así como factores sociodemográficos y culturales que favorecen la elección de la música como vocación profesional. Además, se detectó que los estudiantes de música tienen un antecedente de músicos en casa, ya sea padre, madre o abuelos.

Palabras clave: personalidad, música, estudiantes

\section{Abstract}

This paper examines the relationship between music and his election as vocational and personality factors, cultural and demographic. 86 subjects participated in various subject areas (Music, Psychology, Engineering and Medicine). In all areas measured (depression, suicidal, anxiety, paranoia, obsessive-compulsive disorder, schizophrenia, and addictions), the music students were shown to be more prone for about $10 \%$ of superiority over other students. Thus, through a series of correlations, it was shown that there are personality traits and sociodemographic and cultural factors that favor the choice of music as a professional vocation. In addition, it was found that music students have a history of music at home either parent or grandparent.

Keywords: personality, music, students

\footnotetext{
* Correspondencia: pmlanz@anahuac.mx. Head of Psychology Research Department. Universidad Anáhuac México Norte, Edificio CAIDE 3er. Piso, Avenida Universidad Anáhuac \#46 Col. Lomas Anáhuac, Huixquilucan Edo. de México, México C.P. 52786. Teléfono: +52 (55) 56270210 ext. 8395 Recibido: 25-10-11. Revisión desde: 25-11-11. Aceptado: 29-12-11
}

DOI: $10.5839 / \mathrm{rcnp} .2011 .0602 .09$ 


\section{Introducción}

Los investigadores de la personalidad han dedicado un gran esfuerzo a identificar las formas en que los individuos difieren entre sí. Se han sugerido muchas formas de describir las diferencias individuales. En esencia, se cuenta con la opción de clasificar a la gente en un número limitado de grupos o se puede decidir si las personas varían en gradaciones y así describirlas mencionando qué tanto de las dimensiones básicas poseen.

Dentro de los estudios realizados en torno a la personalidad, un aspecto investigado es acerca de la personalidad de los músicos. El intento más profundo son los estudios realizados por Kemp (1981a, 1981b, 1981c, 1996). Este autor administró varios inventarios de personalidad, incluyendo el de Cattell y el cuestionario 16 PF, a grandes muestras de músicos, incluyendo intérpretes, compositores, estudiantes y maestros, así como también a grupos control de no músicos. Los resultados le llevaron a concluir que existe un núcleo común de rasgos de personalidad que caracterizan al músico en todas las esferas: introversión, emoción (sensibilidad e imaginación) e inteligencia. Kemp sugiere que este "perfil de rasgos centrales" es estable durante el curso de desarrollo de los músicos, desde la niñez hasta la vida profesional; el desarrollo de ciertos tipos de destreza musical en ciertas etapas puede requerir también rasgos adicionales, como la fuerza del superyó y el autocontrol, que reflejan necesidades y demandas temporales.

Kemp (1981a) sugiere que el "perfil de rasgo central" que propone está presente en todos los músicos, tomando como norma el intérprete, y se modifica y complementa con otros rasgos en otras especialidades musicales. Por lo tanto, en el compositor la introversión y la emoción del intérprete se vincula con la independencia, la subjetividad y una baja educación moral.

Otra sugerencia usual es que hay distintos estilos de personalidad asociados con los intérpretes de distintos instrumentos. En la investigación de Kemp los ejecutantes de cuerdas y maderas mostraron niveles mayores de introversión que los de metales y teclados, y que los cantantes. También investigó los estereotipos de personalidad que los músicos atribuyen a los ejecutantes de instrumentos diferentes, concertando una serie de discusiones grupales no estructuradas con músicos de una orquesta sinfónica con sede en Glasgow. En un análisis de una de estas discusiones, más que sistemático es impresionista, no obstante confirma y complementa sus hallazgos precedentes. La mayor polarización que surge es entre los ejecutantes de metales y cuerdas. Los metales describieron las cuerdas como "melindrosas", supersensibles y susceptibles", "sin humor", "apocadas" y "empalagosas", mientras las cuerdas consideraron a los metales como "algo toscos y rústicos", "vulgares", "bufones de la orquesta", "extrovertidos y muy ruidosos". Los ejecutantes de metales se describieron a sí mismos como "un grupo de buenos tipos, honestos, directos, que no se ocupan de tonterías y que son los mejores del mundo"; por contraste, las cuerdas se ven a sí mismas como "individuos que trabajan duramente, concientes, estéticos y sensibles".

Martin (1976) investigó la relación entre los intereses musicales y los factores de personalidad y encontró que los niños con un interés en la música se distinguen por extroversión, ajuste, inteligencia y sensibilidad. En otro estudio, Bell y Cresswell (1984) encontraron que una edad temprana y características específicas de personalidad contribuyen a un aprendizaje de instrumentos musicales; esas características incluyen puntajes altos de inteligencia, vinculación alta con el grupo, autogarantía y estabilidad emocional.

Otra investigación (Dyce \& O'Connor, 1994) sugiere que los músicos tienden a ser más arrogantes, dominantes, extrovertidos, abiertos a la experiencia y neuróticos que los estudiantes universitarios. En este estudio se explica que altos niveles de neuroticismo pueden ser necesarios para un tipo de ejecución musical, porque el neuroticismo contribuye a la tensión mental que a su vez influye en aspectos emocionales de la ejecución.

Por otro lado, Henley (1999) menciona que el interés por la música es cultivado desde experiencias tempranas dentro del hogar (por ejemplo, interés de los padres por la música, oportunidad de asistir a clases de música, entre otras); además, en muchos de los casos uno o los dos padres son músicos y esto es un reforzador poderoso para que crezca en el niño el interés por la música así como para la promoción en él de las habilidades musicales. Slodoba y Davidson (1996) apoyan lo reportado por Henley (1999). Estos autores explican que un mayor conocimiento e interés por la música es el resultado de la exposición a esta dentro del contexto familiar y social.

La presente investigación tuvo como propósito examinar la relación entre la música y su elección como vocación profesional, así como los factores de personalidad, culturales y sociodemográficos.

\section{Material y métodos}

\section{Participantes}

Se seleccionó una muestra no probabilística de 86 jóvenes estudiantes, de los cuales el $58,1 \%$ fueron hombres y el $41,9 \%$ mujeres, con un rango de edad de 18 a 25 años $(M=20.5, D$. E.=1.6). El $34,1 \%$ de los participantes fueron estudiantes de Música, 23,3\% de Psicología, 23,3\% Ingeniería y 18,6\% Medicina.

\section{Instrumentos}

Se elaboró un instrumento autoaplicable, anónimo y estructurado, compuesto por las siguientes áreas: sociodemográfica, antecedentes personales (violencia y familia), habilidades musicales y rasgos de la personalidad. EI instrumento tuvo una confiabilidad total de 0.867 .

\section{Procedimiento}

Se solicitó a estudiantes universitarios de Música, Psicología, Ingeniería y Medicina de tres universidades de México que respondieran el cuestionario autoaplicable realizado para medir los rasgos de personalidad y factores sociodemográficos y culturales que promueven la música como elección vocacional en estudiantes.

\section{Resultados}

En la figura 1 se observa que los estudiantes de música reciben una mayor influencia del padre o madre dedicado a la música que los estudiantes de otras carreras, lo cual podría explicar por 


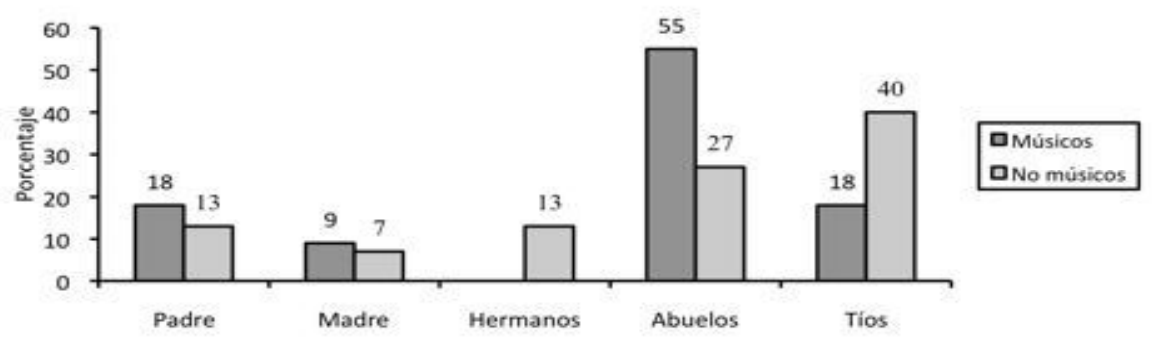

Figura 1. Familiares dedicados a la música de estudiantes músicos y de otras carreras.

Tabla 1. Diferencias en los factores de personalidad entre carreras.

\begin{tabular}{|c|c|c|c|c|c|c|c|c|c|c|}
\hline & \multicolumn{2}{|c|}{ Música $^{1}$} & \multicolumn{2}{|c|}{ Psicología $^{2}$} & \multicolumn{2}{|c|}{ Ingeniería $^{3}$} & \multicolumn{2}{|c|}{ Medicina $^{4}$} & \multirow[b]{2}{*}{$F$} & \multirow[b]{2}{*}{ Post hoc } \\
\hline & $M$ & D. E. & $M$ & D. E. & $M$ & D. E. & $M$ & D. E. & & \\
\hline \multirow{5}{*}{ Violencia } & \multirow{5}{*}{17.8} & \multirow{5}{*}{3.4} & \multirow{5}{*}{17.0} & \multirow{5}{*}{1.7} & \multirow{5}{*}{15.0} & \multirow{5}{*}{0.0} & \multirow{5}{*}{15.0} & \multirow{5}{*}{0.0} & \multirow{5}{*}{$9.50 * *$} & $1=2$ \\
\hline & & & & & & & & & & $1>3,4$ \\
\hline & & & & & & & & & & $2>3$ \\
\hline & & & & & & & & & & $2=4$ \\
\hline & & & & & & & & & & $3=4$ \\
\hline \multirow{3}{*}{ Depresión } & \multirow{3}{*}{24.7} & \multirow{3}{*}{4.3} & \multirow{3}{*}{22.8} & \multirow{3}{*}{2.0} & \multirow{3}{*}{21.4} & \multirow{3}{*}{1.4} & \multirow{3}{*}{21.7} & \multirow{3}{*}{1.3} & \multirow{3}{*}{$6.41^{* *}$} & $1=2$ \\
\hline & & & & & & & & & & $1>3,4$ \\
\hline & & & & & & & & & & $\begin{array}{l}2=1,3,4 \\
3=4\end{array}$ \\
\hline Suicidio & 4.5 & 1.1 & 4.0 & 0.0 & 4.4 & 0.5 & 4.0 & 0.0 & $3.42 *$ & ns \\
\hline Alcoholismo & 24.8 & 4.8 & 22.4 & 0.8 & 25.4 & 4.0 & 24.0 & 3.6 & 2.43 & \\
\hline Ansiedad & 18.9 & 4.3 & 18.8 & 2.8 & 19.6 & 3.1 & 17.7 & 0.8 & 0.97 & \\
\hline Fobia social & 4.3 & 0.8 & 4.2 & 0.4 & 4.8 & 1.6 & 4.5 & 0.5 & 1.47 & \\
\hline Obsesivo-compulsivo & 7.14 & 2.0 & 6.6 & 0.8 & 7.2 & 1.7 & 6.5 & 0.9 & 1.10 & \\
\hline Psicosis & 8.1 & 2.0 & 7.8 & 1.2 & 7.0 & 0.0 & 7.2 & 0.5 & 2.20 & \\
\hline Abuso psicoactivos & 16.4 & 2.9 & 15.0 & 0.0 & 15.0 & 0.0 & 15.5 & 0.5 & $3.67^{*}$ & ns \\
\hline
\end{tabular}

qué hay un porcentaje elevado de abuelos músicos; sin embargo, los resultados mostraron que los estudiantes de música no reportaron tener hermanos dedicados a esta.

Para determinar las diferencias en los factores de personalidad entre los estudiantes de las diferentes carreras se realizaron análisis de varianza de una vía (ver tabla 1). Como se puede observar, se encontraron diferencias significativas en las dimensiones de violencia, depresión, suicidio y abuso de psicoactivos.

Para determinar las diferencias entre los estudiantes de las diferentes carreras, se llevaron a cabo pruebas Post hoc Scheffé, las cuales mostraron que, en el caso de la violencia, los estudiantes de Música presentaron mayores puntajes de esta en comparación con los de Ingeniería y Medicina; sin embargo, no fueron significativamente diferentes de los estudiantes de Psicología, quienes a su vez fueron diferentes a los estudiantes de Ingeniería, donde los de Psicología obtuvieron mayores puntajes en violencia que los de Ingeniería.

En cuanto a la depresión, los resultados mostraron que los jóvenes estudiantes de Música presentaron mayores puntajes que los de Ingeniería y Medicina, pero obtuvieron puntajes similares a los de Psicología. No se encontraron diferencias significativas entre los estudiantes de Psicología, Ingeniería y Medicina.

Cabe señalar que, a pesar de que el análisis de varianza mostró diferencias significativas en las dimensiones de suicidio y de abuso de psicoactivos, las pruebas Post hoc no mostraron diferencias estadísticamente significativas entre los cuatro grupos.
Con el fin de analizar las relaciones entre las variables de rasgos de personalidad y carrera de estudio, así como para describir las relaciones entre las categorías de cada variable, se llevaron a cabo análisis de correspondencia entre rasgos de personalidad y carreras. Cabe mencionar que los análisis se realizaron en algunos rasgos de personalidad utilizando los niveles de sintomatología y en otros con los puntajes de las escalas, dado que no en todos los rasgos se reportaron casos en los tres niveles de sintomatología.

\section{Psicosis por tipo de carrera}

En este análisis se observa que la Chi cuadrada fue de 11.76, la correlación de .037 ( $p=0.068$ ) y en las dimensiones la primera tuvo un nivel bajo con inercia de 0.92 y el segundo nivel alto con inercia de 0.44 . En las filas se encontró que el punto dominante en la primera dimensión fue el de Psicología y en la segunda dimensión fue el de Música. Ingeniería y Medicina reportan puntuaciones iguales.

En cuanto a las contribuciones de cada categoría a la inercia de cada dimensión, Psicología reporta en la primera dimensión un valor de .643 e Ingeniería .198. En tanto que para la segunda dimensión o factor, Música reporta un valor de .651. Psicología explica el 91,5\% del total. Por lo que respecta a las columnas, en la primera dimensión la contribución se dio en el nivel alto, con 2,011, y en la segunda dimensión fue severo con 2,976, explicando el $99,9 \%$ y el $0 \%$ respectivamente.

En la gráfica de distribución por dimensiones de esta correspondencia de la escala de psicosis (figura 2), se encontró 
que la categoría que más discrimina es la carrera de Música, mientras que Psicología, en un cuadrante, e Ingeniería y Medicina, en otro cuadrante, quedan por debajo del baricentro.

En cuanto a las puntuaciones de la escala, se observa que la clasificación de severo se ubica con Música, lo cual indica que son los estudiantes de esta disciplina los que reportan elevada la presencia de síntomas psicóticos, mientras que los estudiantes de Medicina e Ingeniería se ubican dentro del cuadrante con síntomas bajos y Psicología con el nivel alto.

\section{Abuso de psicoactivos por carrera}

Se encontró que el valor de Chi cuadrada fue de 34.54 ( $p=$ 0.99 ), con una desviación típica de 0.07 , lo que indica que el análisis de correspondencias entre puntajes de consumo de psicotrópicos y carrera es estable, y que la correlación de .449 reporta discriminación entre los grupos.

La relación entre las variables fila y columna en el menor número de dimensiones posibles y con una proporción de inercia que explique los datos, el eje que más contribuye es el de nivel bajo con una inercia total de 0.276 que explica el $68,8 \%$ del total; el segundo tiene una inercia total de .354 que explica el $31,2 \%$ del total. En cuanto a los puntos de fila, en la varianza de la primera dimensión Medicina posee 1,044 de varianza y Psicología e Ingeniería .744, en la segunda Medicina y Música. La contribución explicada es de el 95,9\% para Psicología y Medicina.

En cuanto a las columnas, en la primera dimensión la contribución se encontró en el puntaje 15 con 767, que explica el $98,1 \%$, y en la segunda dimensión fue el puntaje 17 con 2,064, explicando el 1,92\%.

En la figura 3 se observa que la carrera que más discrimina es Medicina, mientras Psicología e Ingeniería se ubican en un cuadrante por debajo del baricentro. En cuanto a las puntuaciones sobre consumo de psicotrópicos, se observa que Medicina se acerca al puntaje 16 y Música, aunque lejana, tiene en el mismo cuadrante puntajes altos de 26 y 20, lo cual indica que los estudiantes de esta disciplina son los que reportan elevado el consumo, mientras que los estudiantes de Psicología e Ingeniería se ubican dentro del cuadrante con consumos bajos.

\section{Ideación suicida por carrera}

Se encontró que el valor de Chi cuadrada fue de 40.13 ( $p=$ 0.001 ), con una desviación típica de 0.08 , lo que indica que el análisis de correspondencias entre síntomas de ideación suicida y carrera es estable, y que la correlación de .174 reporta interrelación entre los grupos, ya que la Chi cuadrada es alta y la significancia baja.

En cuanto a la relación entre las variables fila y columna, el eje que más contribuye es el nivel bajo de sintomatología suicida, con una inercia total de 0.354 , que explica el $75,8 \%$ del total; el segundo tiene una inercia total de .336, que explica el $24,2 \%$ del total.

Respecto de los puntos de fila, en la primera dimensión Ingeniería posee 1.386 de varianza y Música .560 explicando el $99,3 \%$ y el 55,1\% respectivamente. En la segunda dimensión, Música tiene .671 y Psicología y Medicina .644. En cuanto a las columnas, en la primera dimensión la contribución se encontró en el puntaje 5 con 2.331 y en la segunda dimensión fueron los puntajes 6 y 7 con 2.064. El puntaje 5 explica el $97,9 \%$ y el puntaje 4 con inercia .029 el $55,1 \%$ del total de la misma.

En la figura 4 se observa que las carreras que más discriminan son Medicina y Psicología; sin embargo, Música es la que se aleja más del baricentro, mientras que Ingeniería se ubica en un cuadrante por debajo de este. En cuanto a las puntuaciones de ideación suicida, se observa que Medicina y Psicología se acercan al puntaje más bajo (4), el puntaje 5 está con Ingeniería y Música, aunque lejanos tiene en el mismo cuadrante puntajes altos de 6 y 7, lo cual indica que son los estudiantes de esta disciplina los que reportan mayor número de signos y síntomas de ideación suicida.

\section{Ansiedad por carrera}

El valor de Chi cuadrada fue de 58.58 ( $p=0.217$ ), con una desviación típica de 0.08 , lo que indica que el análisis sobre la presencia de sintomatología de ansiedad/angustia y carrera es estable. Se encontró que en la relación entre las variables fila y columna, en el menor número de dimensiones posibles y con una proporción de inercia que explique los datos, el eje que más contribuyó fue el de la dimensión 1 con una inercia total de 0.367, que explica el $53,9 \%$ del total; el segundo tiene una inercia total de .208, que explica el $30.5 \%$ del total.

En cuanto a los puntos de fila, en la primera dimensión Ingeniería posee 1.413 de varianza y Medicina .467. En la segunda dimensión Psicología predomina con 1.163, seguida de Medicina con .679. Sobre los puntos de inercia igualmente Ingeniería tiene el mayor puntaje, explicando el $100 \%$ del total. El análisis sobre los datos de columna reportó que los puntajes de sintomatología sobre ansiedad con mayor contribución en la dimensión 1 son 20 y 25, con puntuaciones de 2.333 y en la segunda, 24 y 18 con puntuaciones de 2.551 y 1.284 respectivamente. Los puntajes 20 y 25 indican la inercias más altas, explicadas ambas con el 99,9\%.

Como se puede apreciar en la figura 5, las carreras que más discriminan son Medicina y Psicología; sin embargo, Música es la que se aleja más del baricentro, mientras que Ingeniería se ubica en un cuadrante por debajo de este. En cuanto a las puntuaciones reportadas para la escala de ansiedad, se encontró que Ingeniería y Psicología son las carreras que se encuentra en el baricentro, mientras que Música e Ingeniería están cerca de él pero bajo el mismo. Medicina y Música se acercan a los puntajes más altos de sintomatología y Psicología e Ingeniería a puntajes medios.

\section{Consumo de alcohol por carrera}

En el análisis aplicado a esta escala se encontró que el valor de Chi cuadrada fue de 73.17 ( $p=0.07$ ), con una desviación típica de 0.07 , lo que indica que el análisis de correspondencias entre puntajes de consumo de alcohol y carrera es estable y que la correlación de .011 reporta discriminación entre los grupos.

Se observa que en la relación entre las variables fila y columna, en el menor número de dimensiones posibles y con una proporción de inercia que explique los datos, el eje que más contribuye es la dimensión 1 con una inercia total de 0.458 , que explica el $53,9 \%$ del total; el segundo tiene una inercia total de .280 , que explica el $32,9 \%$ del total. 


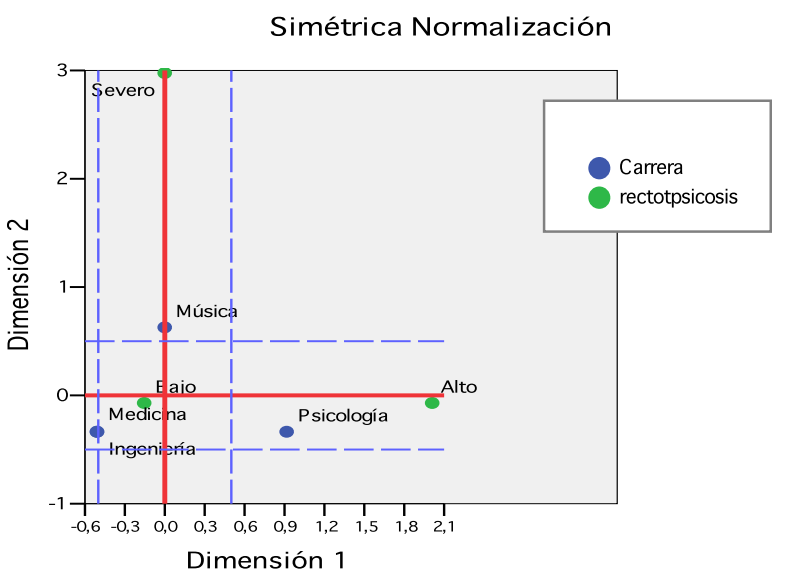

Figura 2. Análisis de correspondencia entre carrera y la escala de psicosis. Puntos de columna y de fila.

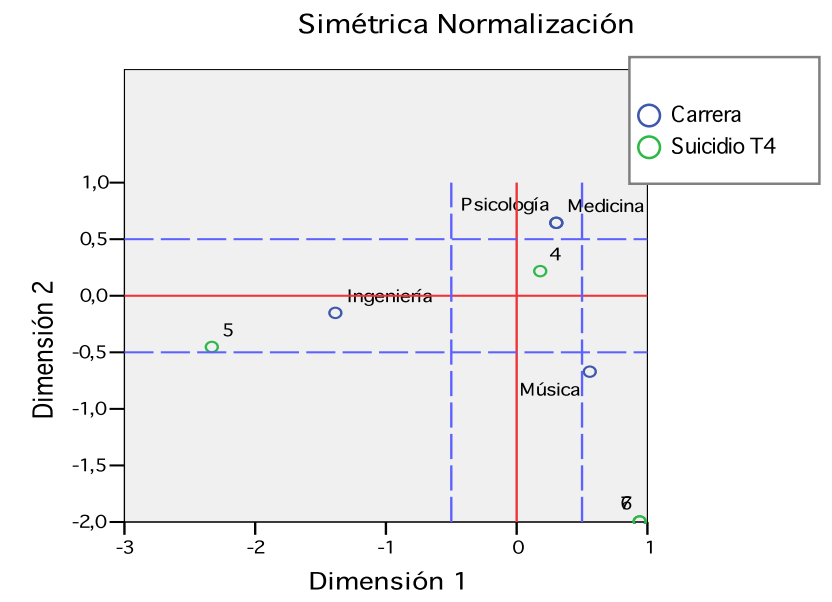

Figura 4. Análisis de correspondencia entre carrera y la escala de ideación suicida. Puntos de columna y de fila.

\section{Simétrica Normalización}

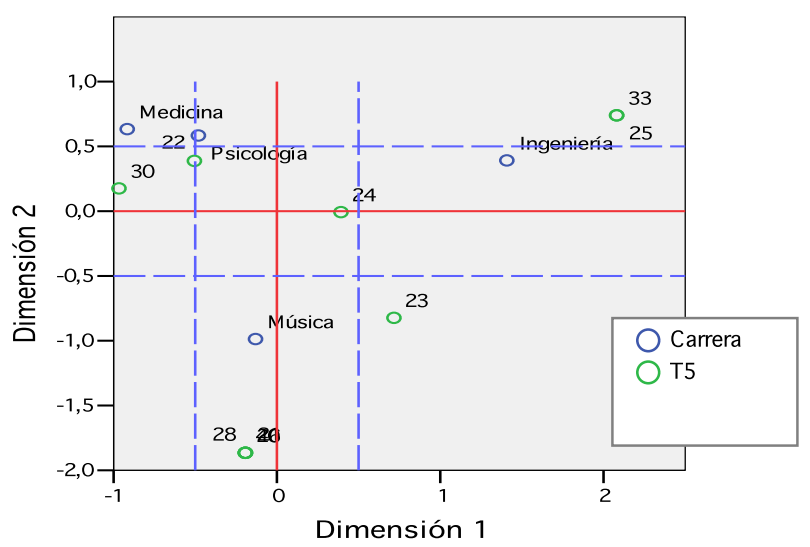

Figura 6. Análisis de correspondencia entre carrera y consumo de alcohol. Puntos de columna y de fila.
Simétrica Normalización

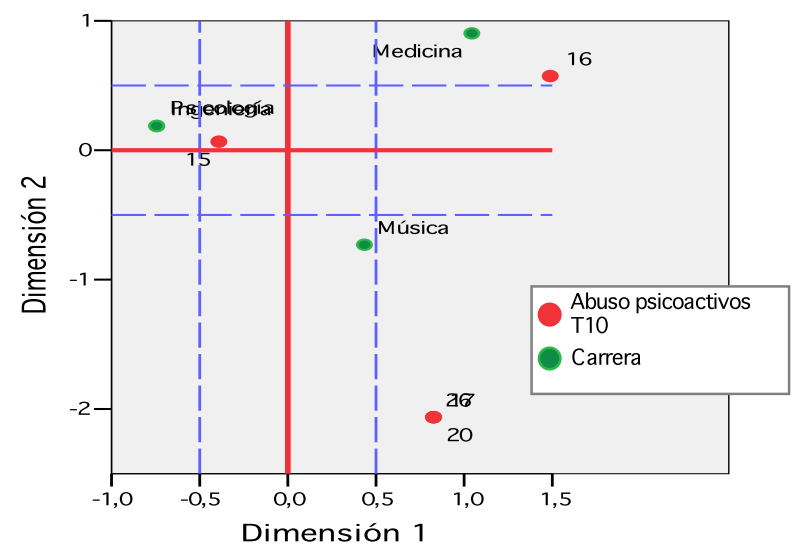

Figura 3. Análisis de correspondencia entre carrera y la escala de abuso de psicoactivos. Puntos de columna y de fila.

\section{Simétrica Normalización}

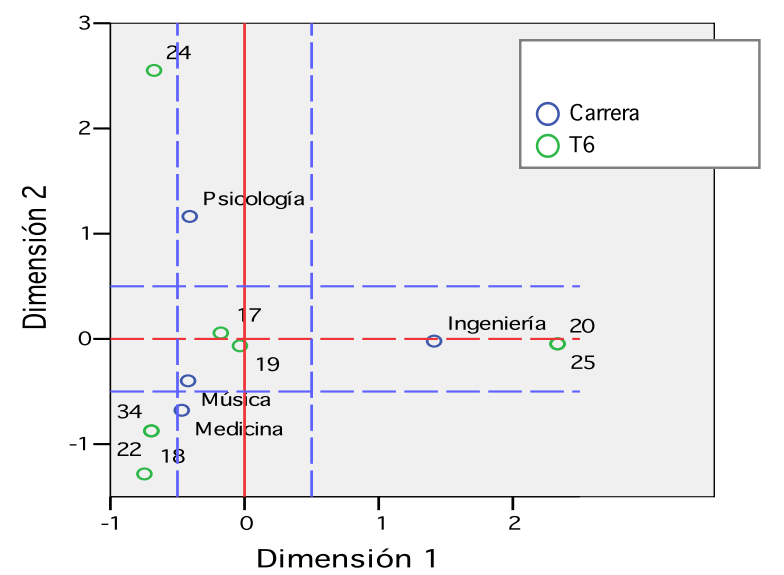

Figura 5. Análisis de correspondencia entre carrera y la escala de ansiedad. Puntos de columna y de fila.

\section{Simétrica Normalización}

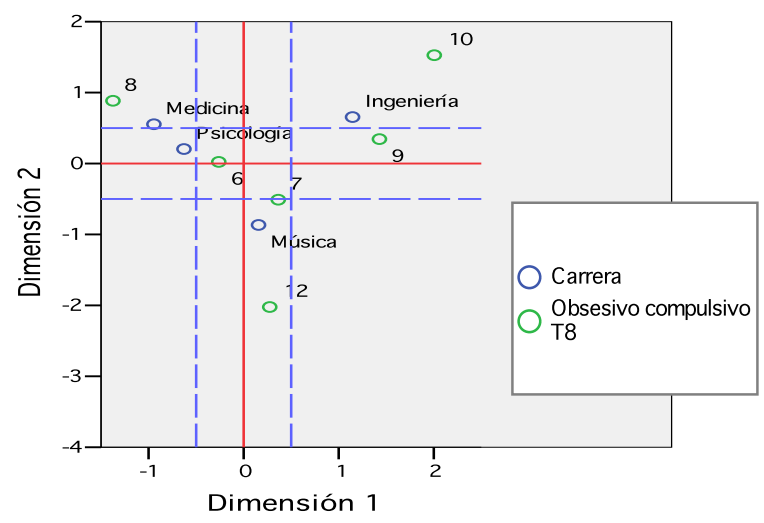

Figura 7. Análisis de correspondencia entre carrera y obsesión-compulsión. 
En cuanto a los puntos de fila, en la primera dimensión Ingeniería posee una puntuación de 1.408 de varianza y Medicina .916, explicando el $93,7 \%$ y el $54,2 \%$ del total respectivamente. En la segunda dimensión, Música tiene .987 y Medicina .484. En relación con los puntos de columna, se observa que en las puntuaciones de la primera dimensión destacan los puntajes de la escala 25 y 33, con puntuaciones de 2,081 ambas. En la segunda dimensión los puntajes relevantes son $21,26,27$ y 40 , todos con 1,866 .

La inercia de la dimensión está en los puntajes de la escala 25 y 33 , explicando ambos el $88,8 \%$ del total. La distribución por dimensiones muestra que la categoría que más discrimina es Ingeniería, lo cual indica que son los estudiantes que reportan elevada la presencia de alcohol; los estudiantes de Medicina, Ingeniería y Psicología se alejan más del baricentro. Los puntajes dados por la escala indican que Ingeniería reporta los más altos (33 y 25), aunque Psicología y Medicina están cerca de los puntajes 30 y 22, y Música 28,26, y 23 (ver figura 6).

\section{Obsesión-compulsión por carrera}

Se encontró que el valor de Chi cuadrada fue de 45.46 ( $p=$ 0.001 ). En la relación entre las variables fila y columna, en el menor número de dimensiones posibles y con una proporción de inercia que explique los datos, el eje que más contribuyó fue el primero, con una inercia total de de 0.327, que explica el $61,7 \%$ del total; el segundo tiene una inercia total de .429 , que explica el $34,7 \%$ del total, y el tercero una inercia de 0.137 , que explica el 3,5\% del total. La desviación típica fue de 0.06 , lo que indica que el análisis de correspondencias es estable y la correlación de 0.449 , reportando discriminación entre los grupos.

Respecto de los puntos de fila, Ingeniería fue el punto dominante en la primera dimensión con 1.147 puntos, y en la segunda dimensión fue Música con .867. La contribución de la inercia a la primera dimensión resultó relevante para Ingeniería, con .535 , y para Medicina, con .290 , explicando el $80,4 \%$ y el $74,9 \%$ de la inercia total respectivamente. Música con un valor de .867 e Ingeniería con uno de .654 fueron relevantes para la segunda categoría (figura 7).

La categoría que más discrimina es Ingeniería lo cual indica que son los estudiantes que reportan alto el rasgo obsesivo compulsivo, mientras que los estudiantes de Medicina y Música son los que se alejan más del baricentro. Las categorías que menos discriminan son las que se encuentran próximas al origen del espacio bidimensional, que son Medicina y Psicología.

\section{Discusión}

La música es el arte de combinar los sonidos de un modo agradable, lo cual, de acuerdo con la literatura, tiene una estrecha relación con los sentimientos. Por otro lado, la personalidad puede definirse como las causas internas que subyacen al comportamiento individual y a la experiencia de la persona. Para algunas corrientes psicológicas la música existe y permanece porque le gente la encuentra recompensante. Para otras es una más de las diversas actividades para pensar que puede realizar el niño, la relación entre música y sociedad es evidente y las diferentes formas de música son objetiva y claramente identificables.
Los resultados del presente estudio, mostraron que existen rasgos de personalidad así como factores sociodemográficos y culturales que favorecen la elección de la música como vocación profesional. Se encontró que los estudiantes de Música tienen un antecedente de músicos en casa ya sea padre, madre o abuelos y que éste es un factor relevante en la elección de la música como profesión.

En el curso del estudio, se observó cómo los estudiantes de música son más propensos a presentar síntomas y signos que los psicólogos, ingenieros o médicos. Es relevante destacar que en la literatura se marca un perfil del estudiante de Medicina como más propenso a la sintomatología en general, mientras que en el presente estudio se obtuvo que son los menos propensos, incluso menos que psicólogos e ingenieros.

En lo que se refiere a las características clínicas que se evaluaron: alcoholismo, consumo de drogas, depresión, ansiedad, ideación suicida, psicosis y obsesión compulsión, los estudiantes de Música reportan en todas ellas porcentajes superiores de presencia de síntomas y signos y, por tanto, de probabilidad de padecer el trastorno.

Con base en los análisis estadísticos realizados se puede concluir que en la muestra estudiada sí existen rasgos de personalidad característicos de los individuos que eligen la música como vocación profesional.

Finalmente, se observó, en el área de adicciones, que los estudiantes de Música tienden a recurrir más a alcohol o drogas que los otros estudiantes. El consumo en general, en las cuatro disciplinas, es elevado; no se reportó consumo moderado de alcohol, los 86 sujetos contestaron que su consumo es de niveles alto a severo, siendo este un dato preocupante.

\section{Referencias}

Dyce, J. A. \& O'Connor, B. P. (1994). The personalities of popular musicians. Psychology of Music, 22(2), 168-173.

Henley, W. R. (1999). The Musician's personality. Creative Research Journal, 12(4), 241-250.

Kemp, A. E. (1981a). Personality differences between the players of string, woodwind, brass and keyboard instruments, and singers. Council for Research in Music Education Bulletin, 66/67, 33-38.

Kemp, A. E. (1981b). The personality structure of the musician. I. Identifying a profile of traits for the performance. Psychology of Music, 9(1), 3-14.

Kemp, A. E. (1981c). The personality structure of the musician. II. Identifying a profile of traits for the composer. Psychology of Music, 9(2), 69-75.

Kemp, A. E. (1996). The musical temperament. Psychology and personality of musicians. Inglaterra: Oxford University Press.

Martin, P. J. (1976). Appreciation of music in relation to personality factors. Tesis Doctoral, no publicada. University of Glasgow, Glasgow, Scotland.

Slodoba, J. \& Davidson, J. (1996). The young performing musician. In I. Deliege \& J. Sloboda (eds.) Musical Beginnings: Origins and development of musical competence. Oxford: Oxford University Press. 